\title{
Document Version Relationship Priority Number
}

National Cancer Institute

\section{Source}

National Cancer Institute. Document Version Relationship Priority Number. NCI

Thesaurus. Code C95361.

An integer specifying the relative rank of a document in comparison to similar documents having the same type of association to a source document. 